\title{
A stable DNA-free screening system for CRISPR/RNPs-mediated gene editing in hot and sweet cultivars of Capsicum annuum
}

\author{
Hyeran $\mathrm{Kim}^{*}{ }^{*}$, Jisun $\mathrm{Choi}^{\dagger}$ and Kang-Hee $\mathrm{Won}^{\dagger}$
}

\begin{abstract}
Background: DNA-free, clustered regularly interspaced short palindromic repeats (CRISPR)-associated protein (Cas) ribonucleoprotein (RNP)-based genome editing is a simple, convincing, and promising tool for precision crop breeding. The efficacy of designed CRISPR-based genome editing tools is a critical prerequisite for successful precision gene editing in crops.

Results: This study demonstrates that soil-grown leaf- or callus-derived pepper protoplasts are a useful system for screening of efficient guide RNAs for CRISPR/Cas9 or CRISPR/Cas12a (Cpf1). CRISPR/Cas9 or Cpf1 were delivered as CRISPR/RNP complexes of purified endonucleases mixed with the designed single guide RNA, which can edit the target gene, CaMLO2 in two pepper cultivars with whole genome sequenced, Capsicum annuum 'CM334' and C. annuum 'Dempsey'. The designed guide RNAs (sgRNAs for Cas9 or crRNAs for Cpf1) are conserved for CaMLO2 in both CM334 and Dempsey and cleave CaMLO2 in vitro. CRISPR/Cas9- or /Cpf1-RNP complexes were transfected into purely isolated protoplasts of the hot pepper CM334 and sweet pepper Dempsey by PEG-mediated delivery. Targeted deep sequencing analysis indicated that the targeted CaMLO2 gene was differentially edited in both cultivars, depending on the applied CRISPR/RNPs.

Conclusions: Pepper protoplast-based CRISPR guide-RNA selection is a robust method to check the efficacy of designed CRISPR tools and is a prerequisite for regenerating edited plants, which is a critical time-limiting procedure. The rapid and convincing selection of guide RNA against a target genome reduces the laborious efforts for tissue culture and facilitates effective gene editing for pepper improvement.
\end{abstract}

Keywords: Pepper genome editing, Capsicum annuum CM334, C. annuum Dempsey, CRISPR/Cas9, CRISPR/LbCpf1, Pepper leaf protoplasts, Pepper callus protoplasts

\section{Background}

Clustered regularly interspaced short palindromic repeats (CRISPR)-CRISPR-associated protein (Cas), CRISPR/Cas9 has emerged as the first RNA-guided genome-editing tool to introduce a target mutation in any sequenced genome after being reported as a programmable molecular scissor in 2012 [1]. Since obtaining SpCas9-based CRISPR tools

\footnotetext{
* Correspondence: ranny@kangwon.ac.kr

${ }^{\dagger}$ Jisun Choi and Kang-Hee Won contributed equally to this work. Department of Biological Sciences, Kangwon National University, Kangwondaehak-gil 1, Chuncheon 24341, South Korea
}

from Streptococcus pyogenes, various tools have been developed from different strains, such as Staphylococcus aureus Cas9 [2], Francisella novicida Cas9 [3], Streptococcus thermophilus Cas9 [4], and Campylobacter jejuni Cas9 [5]. These developed CRISPR-based tools have been promptly applied to all kinds of research areas from generating knock-out cell lines and organisms to biotechnology of animals [6, 7], plants [8-11], and humans [12-14].

CRISPR/Cas12a (Cpf1) has been harnessed for another useful RNA-guided genome editing tool, comprising a single crRNA and a Cpf1 protein that functions in

(c) The Author(s). 2020 Open Access This article is licensed under a Creative Commons Attribution 4.0 International License, which permits use, sharing, adaptation, distribution and reproduction in any medium or format, as long as you give appropriate credit to the original author(s) and the source, provide a link to the Creative Commons licence, and indicate if changes were made. The images or other third party material in this article are included in the article's Creative Commons licence, unless indicated otherwise in a credit line to the material. If material is not included in the article's Creative Commons licence and your intended use is not permitted by statutory regulation or exceeds the permitted use, you will need to obtain permission directly from the copyright holder. To view a copy of this licence, visit http://creativecommons.org/licenses/by/4.0/ The Creative Commons Public Domain Dedication waiver (http://creativecommons.org/publicdomain/zero/1.0/) applies to the data made available in this article, unless otherwise stated in a credit line to the data. 
crRNA processing, target-site recognition, and DNA cleavage [15]. Multiple Cpf1 proteins were obtained from various strains, including FnCpf1 from Francisella tularensis subsp. novicida U112 [16], LbCpf1 from Lachnospiraceae bacterium ND 2006, and AsCpf1 from Acidaminococcus sp. BV3L6 [15]. Both LbCpf1 and AsCpf1 tools showed successful editing activity in human cells by expression plasmids [15] or Cpf1-ribonucleoprotein (RNP) [17]. The editing effect of both LbCpf1 and AsCpf1 was also successfully validated in soybean and tobacco via Cpf1-RNP [18]. However, plant-specific properties lowered the editing efficiency of mature crRNA-harboring plasmids in rice [19] as well as in soybean and tobacco [18].

To successfully improve target gene editing without any off-target mutation, high-fidelity versions of the Cas9 protein were devised using protein engineering [20, 21]. A guide-RNA format was designed using truncated guide RNA for Cas9 [22], chemically synthesized guide RNA for Cpf1 protein [18, 22], or detoxifying format as a $5^{\prime}$-hydroxyl guide RNA for both Cas9 and Cpf1 [23]. Various delivery methods for CRISPR tools to target organisms are available, including mechanical force-, chemical-, and biological system-based methods. In plants, bombardment [11], Polyethylene glycol (PEG)-mediated [24], and Agrobacterium-mediated [25] applications are used to deliver the designed CRISPR tools.

Capsicum (pepper) from the family Solanaceae is known to be recalcitrant to genetic manipulation, such as transformation of a target gene and generation of mutant plants. An efficient method for reverse genetic studies in this genus is still lacking although particle bombardment or Agrobacterium-mediated transformation has been extensively tested for more than 30 years and similar methods have been developed for other genera of the Solanaceae such as Solanum including tomato [26, 27] and Nicotiana (tobacco) [28, 29]. Moreover, CRISPR-based genome editing tools have not been reported in pepper.

C. annuum 'CM334' and 'Dempsey' are excellent resources for studying the traits of hot peppers and sweet peppers, respectively, because of their complete genome information [30, 31]. Unlike protoplast systems from model plants such as Arabidopsis, tobacco, and rice, which have been extensively used for cell-based studies, pepper protoplasts are prone to collapse due to their sticky property after protoplast isolation. Recently, we successfully induced and maintained pepper-derived calluses from soil-grown leaves of two peppers, the hot pepper CM334 and the sweet pepper Dempsey [32]. These calluses support stable pepper protoplasts to characterize cell-based, functional genetic studies on hot and sweet peppers.

Powdery mildew is a significant fungal disease for greenhouse- and field-grown crops such as tomato and pepper [33, 34]. Among the mildew resistance locus $O$ $(M L O)$ genes in plants, AtMLO2 belongs to clade $\mathrm{V}$ along with AtMLO6 and AtMLO12, and it is a wellknown susceptibility gene that confers broad-spectrum resistance in the null mutant against plant pathogens, especially against powdery mildew $[35,36]$. The sequenced genome of C. annuum 'CM334' presumably contains 18 members of CaMLO (Table 1). Previously, CaMLO2, an ortholog of AtMLO2, was reported as a susceptible gene in peppers against biotrophic and hemibiotrophic pathogens $[37,38]$. Due to a lack of targeted mutagenesis in peppers, there is no available pepper $C a M L O$ knockout mutant, except for the natural variants.

Here, we present a DNA-free, genome-editing method in two pepper cultivars, C. annuum 'CM334' and 'Dempsey', using preassembled SpCas9 or LbCpf1 with a single guide RNA RNP, CRISPR/Cas9-RNP or CRISPR/ LbCpf1-RNP, respectively. To test whether CRISPR-RNP tools can be screened in cellular systems of two peppers, we delivered CRISPR/Cas9-RNPs or LbCpf1-RNPs to pepper protoplasts isolated from soil grown Dempsey leaf and proliferative CM334 callus and analysed insertion and deletion (indel) frequencies and patterns at the target $\mathrm{CaMLO} 2$ gene. Pepper protoplast-based guide RNA screening is thus a starting point to evaluate the efficacy of designed CRISPR systems for further investigation of a gene of interest in the generation of stable transgenic peppers.

\section{Results}

PEG-mediated CRISPR-RNP delivery in pepper protoplasts To assess whether CRISPR-RNPs can be delivered to protoplasts of CM334 and Dempsey, we isolated protoplasts from the two pepper cultivars grown in soil (Fig. 1a and b). Dempsey leaf protoplasts were stable enough to be applied in CRISPR-RNPs. In contrast, the CM334 leaf protoplast isolates, although pure (Fig. 1b), were unstable and challenging to harvest after delivery of CRIS PR/Cas9-RNP. Previously, we established leaf-derived calluses from soil-grown CM334 and Dempsey, which can provide stable protoplasts for cell-based studies [32]. We thus explored callus-derived pepper protoplasts as a platform for screening an appropriate gene editing tool (Fig. 1c and d).

\section{In vitro validation of the designed CRISPR RNPs for Cas9 and LbCpf1}

The genomic region of the CaMLO2 gene in both CM334 and Dempsey was firstly analyzed by Sanger sequencing to confirm the conserved exon sequences. We subsequently designed two sgRNAs on the 3rd exon for Cas9 proteins (sgRNA1: 5'-ACATCTTCATCTGCCT TACA-3' and sgRNA2: 5' TGATGACCCTTGTTTA CAAA- $3^{\prime}$ ) and two crRNAs on the 1st and 3rd exon for 
Table 1 MLO proteins in Arabidopsis thaliana and Capsicum annuum

\begin{tabular}{|c|c|c|c|c|}
\hline Species & Gene name & Accession No. & Gene ID & ID source \\
\hline \multirow[t]{3}{*}{ Arabidopsis thaliana } & AtMLO2 & Q9SXB6 & AT1G11310 & \multirow[t]{3}{*}{ UniProt } \\
\hline & AtMLO6 & Q94KB7 & AT1G61560 & \\
\hline & AtMLO12 & O80961 & AT2G39200 & \\
\hline \multirow[t]{18}{*}{ Capsicum annuum } & CA00g74950 & CA00g74950 & CA00g74950 & \multirow[t]{18}{*}{ Sol Genomics } \\
\hline & CA02g02090 & CA02g02091 & CA02g02092 & \\
\hline & CA02g04140 & CA02g04141 & CA02g04142 & \\
\hline & CA02g20140 & CA02g20141 & CA02g20142 & \\
\hline & CA02g21400 & CA02g21401 & CA02g21402 & \\
\hline & CA06g10510 & CA06g10511 & CA06g10512 & \\
\hline & CA06g10520 & CA06g10521 & CA06g 10522 & \\
\hline & CA06g26150 & CA06g26151 & CA06g26152 & \\
\hline & CA07g17840 & CA07g17841 & CA07g17842 & \\
\hline & CA08g01760 & CA08g01761 & CA08g01762 & \\
\hline & CA08g01780 & CA08g01781 & CA08g01782 & \\
\hline & CA08g05700 & CA08g05701 & CA08g05702 & \\
\hline & CA08g13470 & CA08g13471 & CA08g13472 & \\
\hline & CA09g10750 & CA09g10751 & CA09g10752 & \\
\hline & CA09g10760 & CA09g10761 & CA09g10762 & \\
\hline & CA10g07880 & CA10g07881 & CA10g07882 & \\
\hline & CA11g19200 & CA11g19201 & CA11g19202 & \\
\hline & CA12g10780 & CA12g10781 & CA12g10782 & \\
\hline
\end{tabular}

Eighteen of CaMLO proteins in Capsicum annuum were obtained by the BLAST tool in Sol Genomics Network (https://solgenomics.net/) based on three AtMLO proteins (AtMLO2, AtMLO6, and AtMLO12) as a query protein sequence. BLAST is performed with the default setting in the database (Capsicum annuum cv CM334 Genome protein sequences (release 1.55). Accession No. and Gene ID were retrieved from sequence resource sites (Arabidopsis thaliana, https://www.uniprot.org/; Capsicum annuum, https://solgenomics.net/)

LbCpf1 (crRNA1: 5'-TTGAACAAATTATGCATC ACCTT-3' and crRNA2: 5'-GGGACACATAAGTTAG AAACTGG-3') (Fig. 2a and b). We selected specific guide RNAs without up to two nucleotide mismatches based on the entire homology search against the current pepper reference genome using Cas-Designer from RGEN tools [39]. We performed in vitro cleavage assays to validate the activity of CRISPR-RNP complexes of Cas9-sgRNA and LbCpf1-crRNA, consisting respectively of recombinant Cas9 and LbCpf1 proteins and in vitro transcribed guide RNAs, in two pepper cultivars. The target fragment of CaMLO2 was amplified with a primer pair, F and R, denoted in Fig. 2a and Table 3. Cas9RNPs and LbCpf1-RNPs efficiently cleaved the target regions of CaMLO2 in both CM334 and Dempsey in vitro, as expected (Fig. 2c and d).

Analysis of CRISPR/Cas9-RNP in Dempsey leaf protoplasts We investigated PEG-mediated CRISPR/Cas9 RNP delivery into protoplasts isolated from CM334 and Dempsey leaves. First, we tested whether pepper protoplasts were transiently transfected with a conventional plasmid harboring a GFP:NLS expressing cassette as a nuclear marker. The Dempsey leaf protoplasts were efficiently transfected and showed GFP signals in the nucleus after $24 \mathrm{~h}$ of incubation (Fig. 3a). However, CM334 leaf protoplasts were not stable enough to express the transfected plasmid or CRISPR/Cas9 RNP. Moreover, Dempsey leaf protoplasts were successfully transfected and maintained until the detection of transfected GFP plasmid or subsequent genotype analysis. Dempsey protoplasts transfected with CRISPR/Cas9-RNP were harvested at $24 \mathrm{~h}$ and $48 \mathrm{~h}$ of incubation (Fig. $3 \mathrm{~b}$ ). These were used to extract the genomic DNA and perform targeted deep sequencing to analyze the indel frequencies and patterns at the target sites in the CaMLO2 gene. Indels using CRISPR/Cas9-RNP at $24 \mathrm{~h}$ were marginally captured at the target sites with frequencies of either $1.23 \%$ for sgRNA1 or $0.02 \%$ for sgRNA2 in CaMLO2 at $24 \mathrm{~h}$ (Fig. $3 \mathrm{~b})$. After $48 \mathrm{~h}$ of incubation, indels were dramatically increased and differentially captured at the target sites with $11.3 \%$ for sgRNA1 or $0.5 \%$ for sgRNA2 (Fig. 3b). Most of the indels induced at the $\mathrm{CaMLO} 2$ gene using Cas9-sgRNA1 complexes were deletions of several nucleotides located 3 bp upstream of PAM (as $5^{\prime}$-CCT-3'), compared with Cas9-sgRNA2 (Fig. 3c). This result 


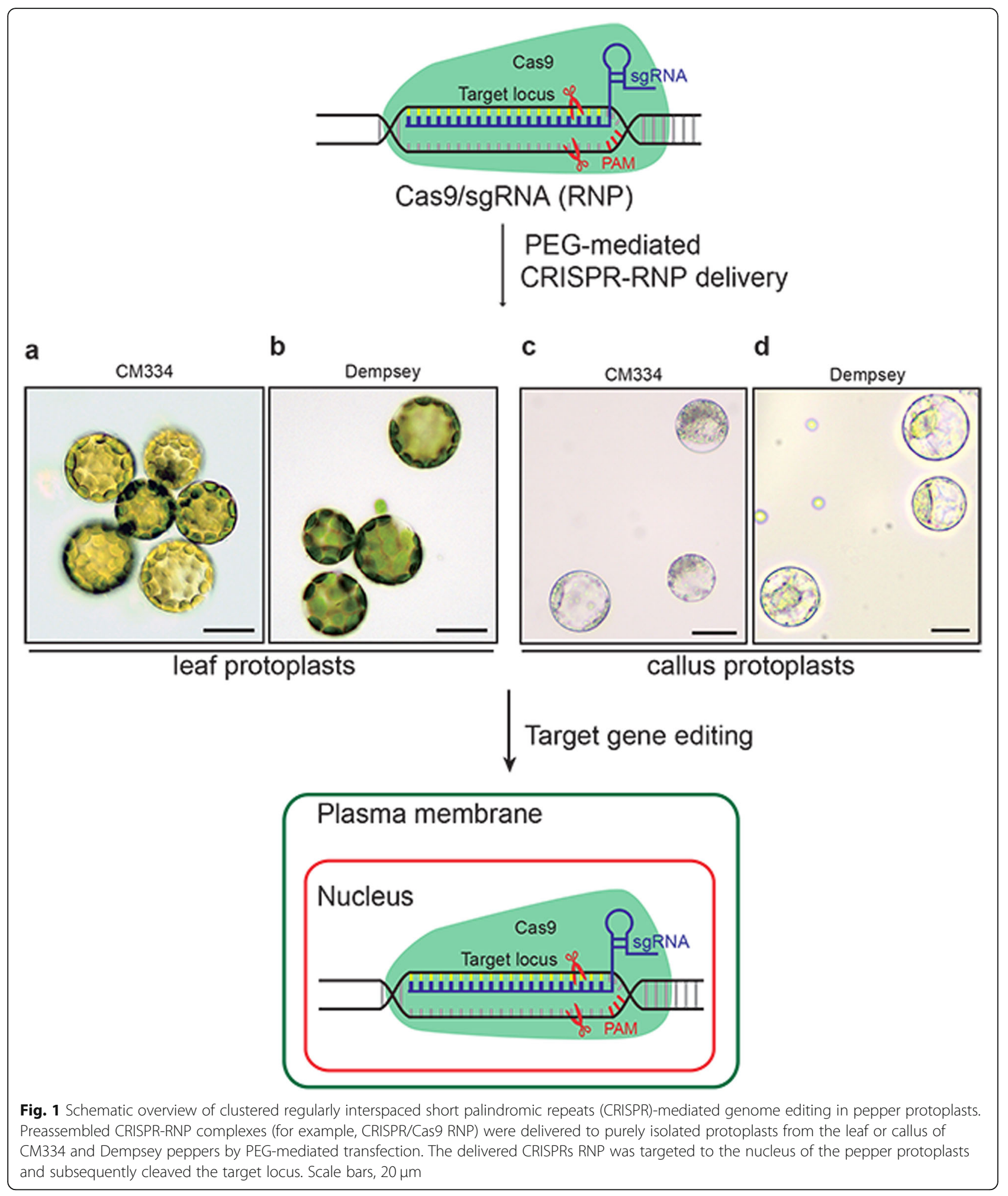

indicated that the designed sgRNA1 containing CRISPR/ Cas9-RNP complex is more effective to edit the CaMLO2 gene than sgRNA2. All results demonstrated that Dempsey leaf protoplasts are a stable cellular system that can be used to validate an efficient CRISPR/Cas9RNP for target gene editing in sweet pepper. 


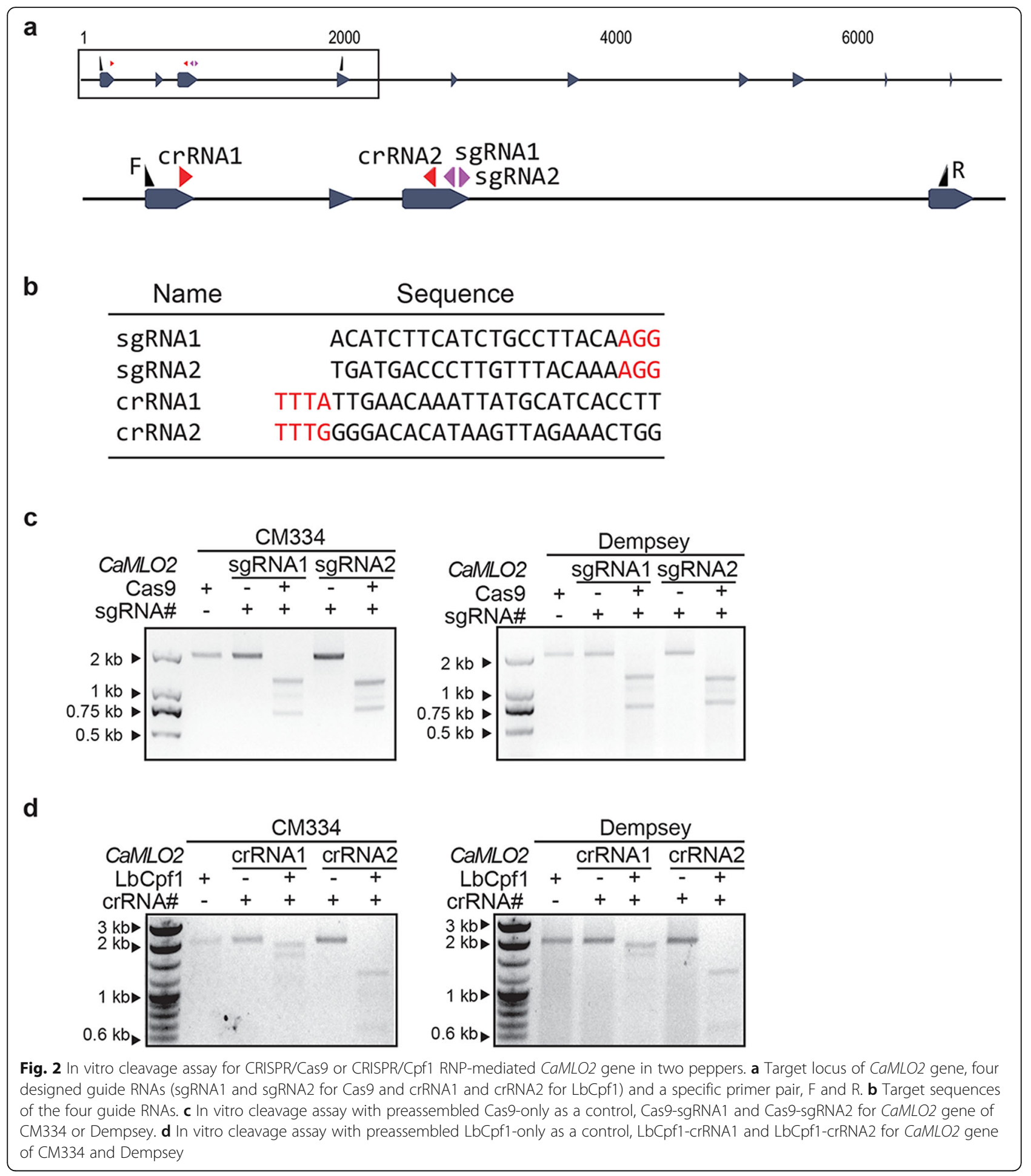

Analysis of CRISPR/Cas9-RNP in CM334 callus protoplasts To explore a cellular system for CM334, we used the established propagating callus lines from CM334 leaves. Pure isolates of CM334 callus-derived protoplasts were obtained (Fig. 1c) and transfected with a plasmid containing the GFP:NLS expressing cassette. The transfected
CM334 callus protoplasts expressed and distinctively demonstrated GFP signals in the nucleus after $48 \mathrm{~h}$ of incubation (Fig. 4a). Therefore, we further explored whether CM334 callus protoplasts carry CRISPR/Cas9RNP and validated the active guide RNAs as a screening system to evaluate CRISPR-RNPs. We delivered the 


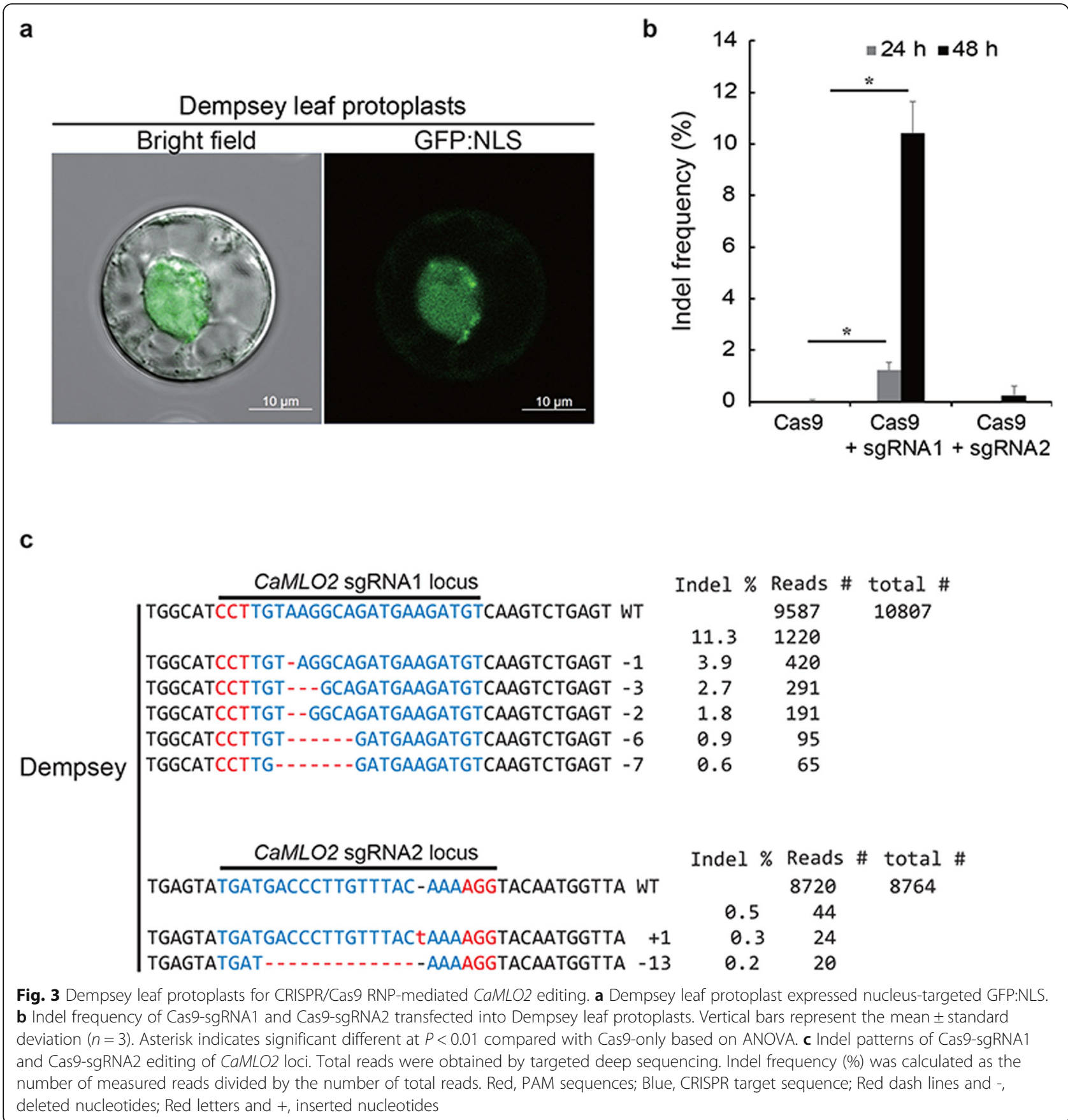

preassembled Cas9 proteins together with the designed sgRNA1 and sgRNA2 as RNP complexes into CM334 callus protoplasts. The transfected CM334 callus protoplasts were incubated for $48 \mathrm{~h}$ before analyzing the editing efficacy. The Cas9-only control did not induce any mutation at the target locus of CaMLO2, whereas the Cas9-sgRNA1 complexes induced $17.6 \%$ of indel mutations at the target site (Fig. 4b and c). However, the activity of Cas9-sgRNA2 complexes (approximately 0.2\%) was less efficient in inducing indel mutations, unlike Cas9-sgRNA1 complexes, despite the sparse indel patterns (Fig. 4b and c). The active Cas9-sgRNA1 complexes mainly conferred deletions of several nucleotides. These results indicate that Cas9-sgRNA1 RNP complexes actively edit $\mathrm{CaMLO} 2$ and could be used in further regeneration procedures to produce CaMLO2 mutations in the hot pepper CM334. 


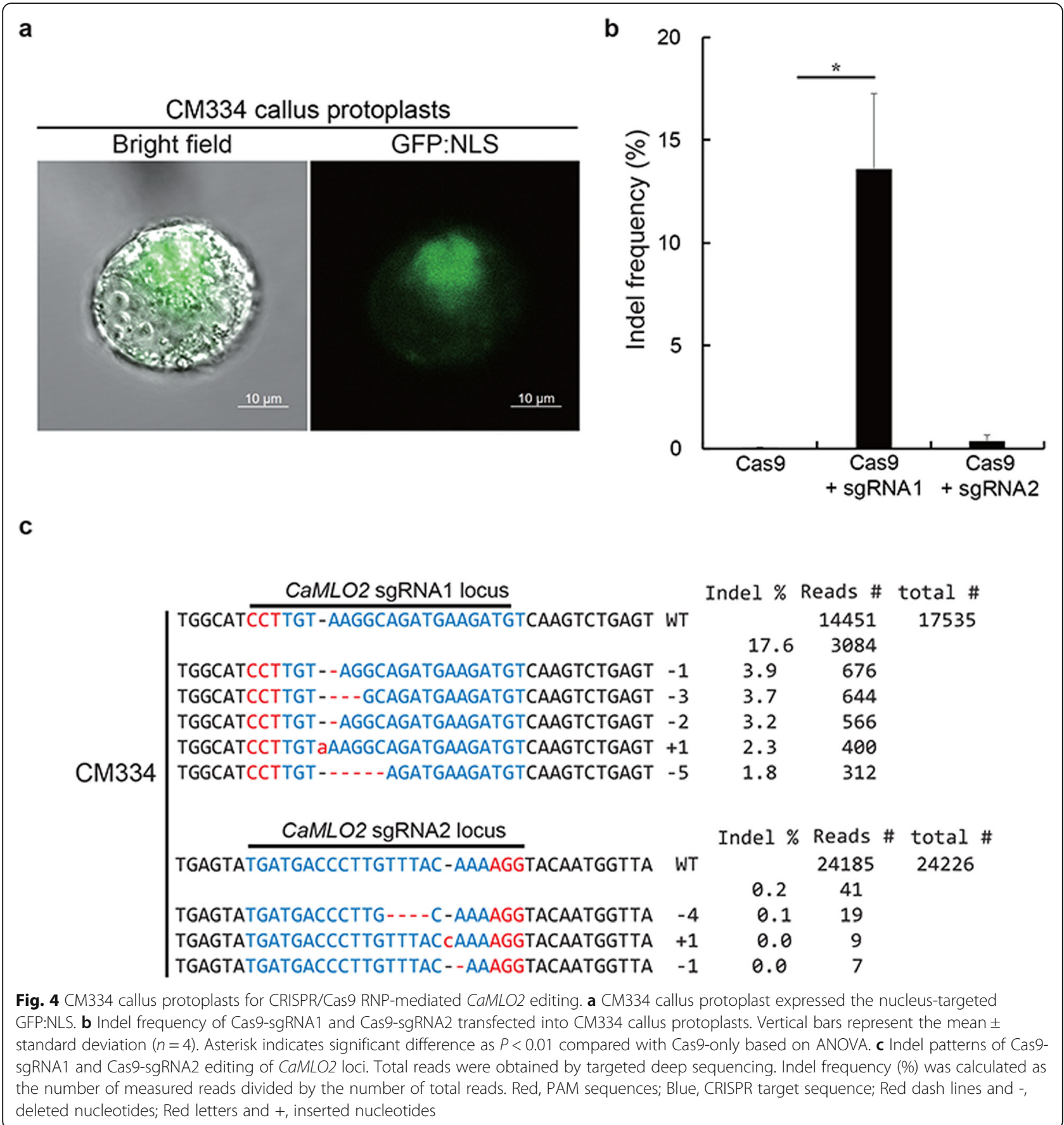

\section{Analysis of CRISPR/LbCpf1-RNP in CM334 callus protoplasts}

We examined the activity of LbCpf1-RNP in CM334 callus protoplasts via PEG-mediated delivery. The complexes of LbCpf1-crRNA1 and LbCpf1-crRNA2 were successfully delivered into CM334 callus protoplasts. The transfected callus protoplasts were stable enough to evaluate indel mutations, unlike the leaf protoplasts. Protoplasts with LbCpf1 only were used as a control for LbCpf1 RNP transformation. The protoplasts transfected with LbCpf1-crRNA complexes exhibited indel frequencies of 9.9\% for LbCpf1-crRNA1 and 19.3\% for LbCpf1crRNA2 (Fig. 5a). The designed LbCpf1-crRNA2 activity was two-fold higher than that of LbCpf1-crRNA1 based on the induced indel frequencies at the CaMLO2 gene loci. As previously reported with the distinct activities of designed guide RNAs in soybean, cabbage, and petunia $[18,40,41]$, the two designed crRNAs of CaMLO2 exhibited differential editing efficacy based on unknown properties of the sequence context of a target gene. 


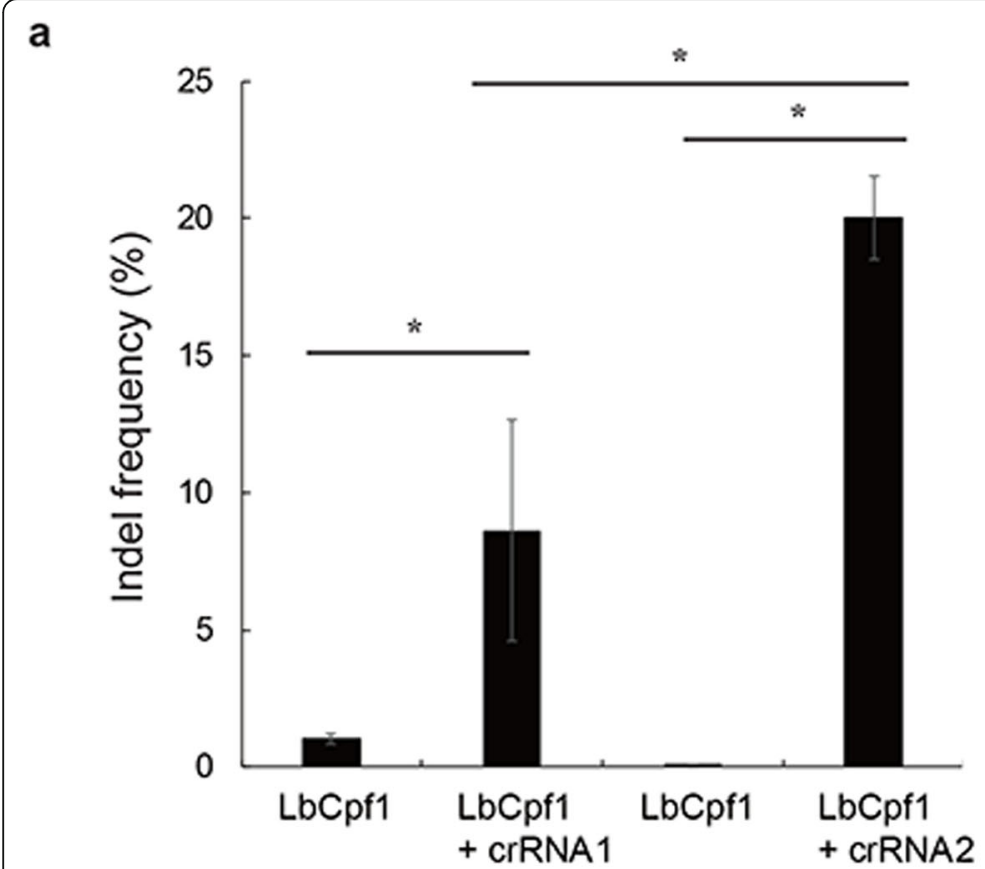

b

\begin{tabular}{|c|c|c|c|c|c|}
\hline & CaMLO2 crRNA1 locus & \multicolumn{2}{|c|}{ Indel \% } & eads \# & total \# \\
\hline & CCATTTTTATTGAACAAATTATGCATCACCTTGGAGAGGT & WT & & 18045 & 20020 \\
\hline & & & 9.9 & 1975 & \\
\hline & CCATTTTTATTGAACAAATTATG - - - - CTTGGAGAGGT & -6 & 1.6 & 318 & \\
\hline M334 & CCATTTTTATTGAACAAATTATGCA - - - - TGGAGAGGT & -6 & 0.6 & 119 & \\
\hline & CCATTTTTATTGAACAAATTATGCA - - - - TTGGAGAGGT & -5 & 0.5 & 106 & \\
\hline & CCATTTTTATTGAACAAATTA- & -10 & 0.4 & 87 & \\
\hline & CCATTTTTATTGAACAAATTATG - - - - - CCTTGGAGAGGT & -5 & 0.4 & 86 & \\
\hline
\end{tabular}

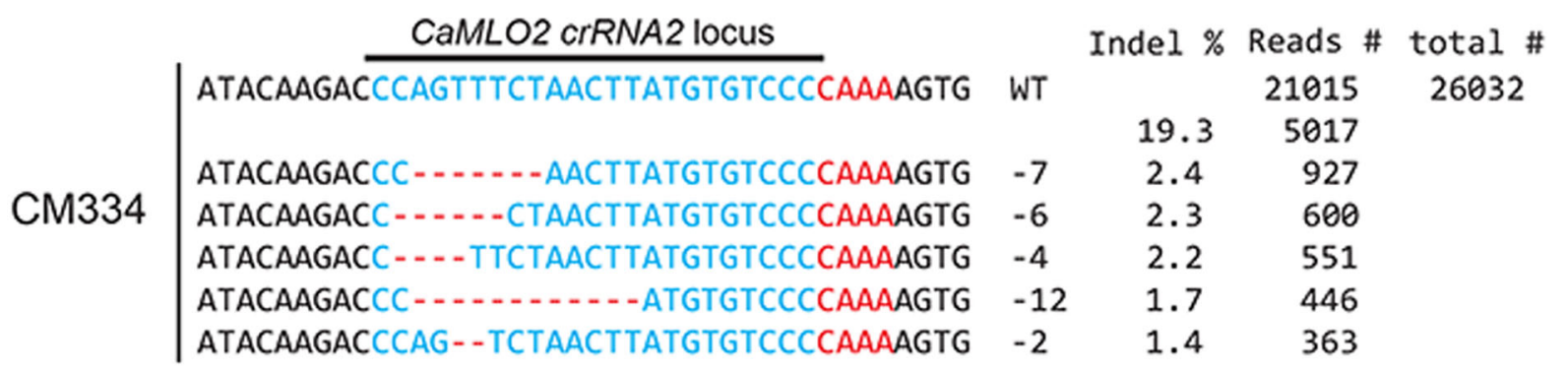

Fig. 5 CM334 callus protoplasts for CRISPR/LbCpf1 RNP-mediated CaMLO2 editing. a Indel frequency of LbCpf1-crRNA1 and LbCpf1-crRNA2 transfected into CM334 callus protoplasts at $48 \mathrm{~h}$ post-incubation. Vertical bars represent the mean \pm standard deviation $(n=3)$. The asterisk indicates significant difference as $P<0.01$ compared with LbCpf1 only based on ANOVA. b Indel patterns of LbCpf1-crRNA1 and LbCpf1-crRNA2 editing of CaMLO2 loci. Total reads were obtained by targeted deep sequencing. Indel frequency (\%) was calculated as the number of measured reads divided by the number of total reads. Red, PAM sequences of TTTN; Blue, CRISPR target sequence; Red dash lines and -, deleted nucleotides

The indel patterns of targeted LbCpf1-crRNA1 complexes varied at the CaMLO2 gene locus in CM334 callus protoplasts, with several deletions of nucleotides $(-6,-5$, and -10$)$ in the first five ranked reads (Fig. 5b).
The other targeted LbCpf1-crRNA2 complexes also showed varied indel patterns with distinct deletions of nucleotides $(-7,-6,-4,-12$, and -2$)$ for CaMLO2 crRNA2 locus in the first five ranked reads (Fig. 5b). 
The validated LbCpf1-crRNA2 complexes can be used for $\mathrm{CaMLO} 2$ editing in CM334. These data indicate that the established stable pepper protoplasts are robust systems for screening of effective CRISPR tools that can be utilized for precision editing in peppers.

\section{Discussion}

Here, we demonstrated that Dempsey leaf protoplasts and CM334 callus protoplasts are stable and robust cellbased systems for evaluating the CRISPR tools Cas9 and LbCpf1. Using the designed guide RNAs, sgRNAs for Cas9 and crRNAs for LbCpf1, we can examine whether the applied guide RNAs are active enough to induce gene editing specifically at the target sites. In Dempsey, Cas9-sgRNA1 RNP has an indel frequency of $11.3 \%$. The designed Cas9-sgRNA1 complex can thus be used to generate CaMLO2-edited Dempsey pepper with broadspectrum resistance to powdery mildew [38] and hemibiotrophic bacterial pathogens such as Xanthomonas campestris pv. vesicatoria and Pseudomonas syringae pv. tomato (Pst) DC3000 [37]. However, Cas9-sgRNA2 RNP did not enough induce indel mutation at the locus of sgRNA2 in CaMLO2. Although in silico designed guide RNAs are available throughout a reference genome, the efficacy of the designed guide RNAs needs to be validated in the aimed gene of a target genome. Due to unknown properties such as chromatin structure or epigenetic modification, of the sequence context of a target gene, we frequently observed the differential geneediting efficiency in previous reports $[18,40,41]$. Therefore, a stable screening tool of active CRISPR tool is essential for successful crop editing.

CRISPR/Cas9-RNP in Dempsey leaf protoplasts revealed that the designed Cas9-sgRNA1 complex was 22fold more efficient in editing CaMLO2 compared with the Cas9-sgRNA2 complex. Similarly, Cas9-sgRNA1 RNP complex showed 88-fold higher indel frequency than did Cas9-sRNA2 RNP in CM334 callus protoplasts. The efficacy of the designed sgRNAs for Cas9 to edit CaMLO2 in Dempsey leaf protoplasts was similar to that in CM334 callus protoplasts, although the two peppers were different cultivars. In addition, the indel patterns induced by Cas9-sgRNA1 complexes were similar to the main deletions in both Dempsey and CM334. Undeniably, it is too early to propose a general rule for the efficacy and patterns of guide RNA in pepper genome editing. However, this result raises an interesting question regarding whether a guide RNA designed for a conserved target locus has wide-ranging efficacy among pepper cultivars. This possibility can be systemically investigated at the whole genome scale.

Previously, we reported that another CRISPR-RNP tool, the LbCpf1-crRNA complex, successfully induced indels at two targeted loci of FAD2-1A and FAD2-1B in soybean [18]. Here, we also tested the activity of CRIS PR/LbCpf1-RNP in pepper gene editing. We demonstrated that the efficacy of the designed crRNA1 and cRNA2 for LbCpf1 significantly differed by more than two-fold. This result indicated that CRISPR/LbCpf1RNP precisely and effectively edited the target gene in two peppers.

To act as control experiments for the target specific editing via CRISPR RNPs, we delivered CRISPR proteins without a guide RNA, as either Cas9-only or LbCpf1only, into pepper protoplasts. In the presence of Cas9only for sgRNA1 and sgRNA2, and of LbCpf1-only for crRNA2, there were no induced mutations in the target loci of the CaMLO2 gene. However, LbCpf1-only for crRNA1 locus of CaMLO2 exhibited marginal indel frequency as shown in Fig. 5a. The repeated nucleotide sequences with $13 \mathrm{bp}$ of thymine in the 3 'downstream flanking region of the target crRNA1 locus may have caused the noise in indel frequency (Fig. 5a) and patterns (Fig. S1), unlike the specific indel mutation by CRISPR-crRNA1 complexes (Fig. 5a and b). The indel by LbCpf1-only in the crRNA1 locus was most likely from the error introduced by three consecutive PCR preparations performed to conduct the Next Generation Sequencing (NGS). The LbCpf1 system requires TTTN, T-rich protospacer adjacent motif (PAM) sequence in the 5' upstream of a guide RNA (crRNA). Several in sillico designed crRNAs for LbCpf1 in a target genome are located at AT-rich sites or repeated sequences due to the high chance of PAM properties. Therefore, it is critical to validate whether the aimed mutation was induced at the target locus of the designed crRNA in a target gene.

Furthermore, we revealed that the editing efficacy of active guide RNAs, such as sgRNA1 for Cas9 in Dempsey leaf protoplasts, was cumulative with respect to incubation time in pepper protoplasts. Regarding the editing efficiency for CaMLO2 in CM334, the designed Cas9sgRNA1-RNP is comparable to the tested LbCpf1crRNA2-RNP. The results exhibited the highest indel frequencies of $17.6 \%$ for Cas9-RNP and $19.3 \%$ for LbCpf1-RNP among the tested Cas9 and LbCpf1 RNPs. Thus, either Cas9-sgRNA1 complex or LbCpf1-crRNA2 can be used as a practical tool to edit CaMLO2 in CM334 pepper.

Since the first seminal publications on Arabidopsis, tobacco, and rice in 2013 [8-10], various edited crops were obtained using CRISPR-based tools, including staple foods such as rice [8], wheat [11], soybean [18, 42], and maize [43] as well as vegetables and fruits such as tomato [44], potato [45], watermelon [46], and cabbage [40]. To the best of our knowledge, the present report provides the first data for precise pepper editing in both hot pepper and sweet pepper cultivars. We successfully 
Table 2 Primers used in guide-RNA synthesis

\begin{tabular}{ll}
\hline Primers & Sequence (bold: guide-RNA sequences) \\
\hline sgRNA1-F & GAAATTAATACGACTCACTATAGCATCTTCATCTGCCTTACAGTTTAGAGCTAGAAATAGCAAG \\
sgRNA2-F & GAAATTAATACGACTCACTATAGGATGACCCTTGTTTACAAAGTTAGAGCTAGAAATAGCAAG \\
sgRNA-R & AAAAAAGCACCGACTCGGTGCCACTTITCAAGTTGATAACGGACTAGCCTTATTTAACTTGCTATTTCTAGCTCTAAAAC \\
CrRNA1-F & AAGGTGATGCATAATTTGTTCAAATCTACACTTAGTAGAAATT \\
CrRNA2-F & CCAGTTTCTAACTTATGTGTCCCATCTACACTTAGTAGAAATT \\
CrRNA-R & GAAATTAATACGACTCACTATAGGGATTTCTACTAAGTGTAGAT
\end{tabular}

edited $\mathrm{CaMLO} 2$ genes in the protoplasts of two pepper cultivars with known whole-genome sequences. The established leaf or callus protoplasts are robust systems suitable to explore settled CRISPRs-RNP as well as newly developed genome editing tools for improved pepper traits.

\section{Conclusions}

Designed DNA-free, clustered regularly interspaced short palindromic repeats (CRISPR)/ ribonucleoproteins (RNPs) screening system is a robust and prerequisite tool for precise genome editing in hot and sweet peppers.

\section{Methods}

\section{Plant material and protoplast isolation}

C. annuum cultivars CM334 and Dempsey were provided by the Vegetable Breeding Research Center (VBRC) in Republic of Korea. CM334 and Dempsey were germinated and grown in soil under $16 \mathrm{~h}$ light and $8 \mathrm{~h}$ dark photoperiod at $25 \pm 1{ }^{\circ} \mathrm{C}$ in a growth chamber. Pepper CM334 calluses were produced in a callus inducing media (CIM, MS media contained with B5 vitamins,
$3 \%$ of sucrose, $1 \mathrm{mg} / \mathrm{L}$ 2,4-dichlorophenoxyacetic acid) from fully expanded young leaves and were maintained in CIM by regular subculturing every 3 weeks [32]. The pepper calluses from the two cultivars were digested in a cell wall digesting enzyme solution [47] for $3-4 \mathrm{~h}$ at $24 \pm 1{ }^{\circ} \mathrm{C}$ to isolate the protoplasts. The digested pepper protoplasts were diluted with an equal volume of W5 solution $\left(154 \mathrm{mM} \mathrm{NaCl}, 125 \mathrm{mM} \mathrm{CaCl}_{2}, 5 \mathrm{mM} \mathrm{KCl}, 5 \mathrm{mM}\right.$ glucose, $1.5 \mathrm{mM}$ Mes- $\mathrm{KOH}, \mathrm{pH} 5.6)$ to remove the cell wall digesting enzyme solution. The pepper protoplasts were gently collected by centrifugation at $58 \mathrm{~g}$ for $5 \mathrm{~min}$, and then rinsed with W5 solution two times. The purely isolated pepper protoplasts were counted using a hemocytometer. Approximately $5 \times 10^{4}$ isolated protoplasts were used for PEG-mediated CRISPR-RNPs delivery, as described previously [18] with slight modifications. Briefly, Cas9 or LbCpf1 proteins were premixed with an in silico designed guide RNA as a 1:6 M ratio for $1 \mathrm{~h}$ at room temperature. The preassembled RNP mixtures were carefully suspended with the counted protoplasts in $300 \mu \mathrm{l}$ of MMG solution ( $400 \mathrm{mM}$ mannitol, 15 $\mathrm{mM} \mathrm{MgCl} 2,5 \mathrm{mM}$ MES; pH 5.6). Subsequently, an equal volume of freshly prepared PEG solution (200 mM

Table 3 Primers used in targeted deep sequencing

\begin{tabular}{|c|c|}
\hline Primers & Sequence \\
\hline CaMLO2 F (Fig. 2a) & ATGGCTAAAGAACGGTCGAT \\
\hline CaMLO2 R (Fig. 2a) & ATGGAGCTGGTGTATTGCAT \\
\hline $\begin{array}{l}\text { Primary } F \\
\text { for sgRNA1, sgRNA2, crRNA2 }\end{array}$ & TGGGATTCATATCATTGTTGTTG \\
\hline $\begin{array}{l}\text { Primary } R \\
\text { for sgRNA1, sgRNA2, crRNA2 }\end{array}$ & CCGAATGTGTCTCAGCCTIT \\
\hline Primary $\mathrm{F}$ for crRNA1 & ATGGCTAAAGAACGGTCGAT \\
\hline Primary $\mathrm{R}$ for crRNA1 & GGCACTAAGGTTGGCTACTT \\
\hline $\begin{array}{l}\text { Secondary } F \\
\text { for sgRNA1, sgRNA2, crRNA2 }\end{array}$ & ACACTCTTTCCCTACACGACGCTCTTCCGATCTTGGGATTCATATCATTGTTGTTG \\
\hline $\begin{array}{l}\text { Secondary } R \\
\text { for sgRNA1, sgRNA2, crRNA2 }\end{array}$ & ACTGGAGTTCAGACGTGTGCTCTTCCGATCTCCGAATGTGTCTCAGCCTTT \\
\hline Secondary F for crRNA1 & ACACTCTTTCCCTACACGACGCTCTTCCGATCTATGGCTAAAGAACGGTCGAT \\
\hline Secondary R for crRNA1 & GACTGGAGTTCAGACGTGTGCTCTTCCGATCTGGCACTAAGGTTGGCTACTT \\
\hline
\end{tabular}


mannitol, $100 \mathrm{mM} \mathrm{CaCl}$, 40\% PEG 4000) was added. The PEG-mixed pepper protoplasts were washed three times with an equal volume of serial dilutions using the W5 solution. They were then harvested by centrifugation at $58 \mathrm{~g}$ for $5 \mathrm{~min}$ and subsequently incubated in W5 solution. After incubation for the indicated time (24 or 48 h), CRISPR-RNPs-transfected protoplasts were prepared for genomic DNA extraction and finally analyzed for target gene editing.

\section{Design and preparation of guide RNA}

$\mathrm{CaMLO} 2$ genomic regions from the two cultivars were sequenced by Sanger sequencing to confirm the nucleotide sequences and design the guide RNAs. We used Cas-Designer of RGEN tools (http://rgenome.net) to devise specific candidate sgRNAs for Cas9 and crRNAs for LbCpf1. We obtained two sgRNAs (sgRNA1: 5'-ACAT CTTCATCTGCCTTACA-3', sgRNA2: 5' -TGATGACC CTTGTTTACAAA-3') in the third exon for Cas9 and two crRNAs (crRNA1: 5' -TTGAACAAATTATGCATC ACCTT-3', crRNA2: 5'-GGGACACATAAGTTAGAA ACTGG-3') in the first and third exons for LbCpf1. The guide RNAs were synthesized by in vitro transcription using T7 RNA polymerase (New England Biolabs, MA, USA) as described previously [18, 24]. Primers used for in vitro transcription are listed in Table 2.

\section{In vitro cleavage assays using CRISPR tools}

Pepper genomic DNA was prepared with a Plant SV Mini kit (GeneAll, Seoul, South Korea) and used as a template to amplify the target DNA regions of CaMLO2. The activity of the designed guide RNAs was validated by an in vitro cleavage assay [18]. Briefly, $240 \mathrm{ng}$ of target DNA amplicons were digested with $2 \mu \mathrm{g}$ of purified Cas 9 or LbCpf1 and $1.5 \mu$ g of guide RNA in $2 \mu \mathrm{L}$ of $10 \mathrm{X}$ NEB 3.1 buffer (NEB) for $1.5 \mathrm{~h}$ at $37^{\circ} \mathrm{C}$ and subsequently incubated with RNase A for $1 \mathrm{~h}$ at $37^{\circ} \mathrm{C}$. The digested target DNA was purified using an MG PCR purification SV kit (MGmed, Seoul, South Korea) and analyzed by agarose gel electrophoresis.

\section{Targeted deep sequencing}

The indel frequency and patterns were analyzed by targeted deep sequencing [18]. The prepared pepper genomic DNA was amplified with specific primers (listed in Table 3) for each guide RNA. The target amplicons were attached with multiplexing indices and specific sequencing adaptors by consecutive PCRs and subjected to high-throughput sequencing using Illumina Miseq (V2, 300 cycle) (Macrogen, Seoul, South Korea). The raw data of paired-end Miseq were analyzed using a Cas-Analyzer (http://www.rgenome.net/cas-analyzer/\#!) from the RGEN tools [48].

\section{Statistical analysis}

The data are presented as the means of at least three biological replicates with standard deviation. The significant difference (*, $P<0.01)$ was assessed by one-way ANOVA with post-hoc Tukey HSD.

\section{Supplementary information}

Supplementary information accompanies this paper at https://doi.org/10. 1186/s12870-020-02665-0.

Additional file 1 Fig. S1 Sequence information of CaMLO2 CrRNA1 locus in LbCpf1-only applied CM334 protoplasts. ID, ranking number of sequenced reads by NGS; Yellow, crRNA1 target sequence; Upper sequences within Sequence box, reference genomic sequence; Bottom sequence within Sequence box, sequenced reads using NGS; Misaligned or T, evaluated as deletion or insertion. Note that LbCpf1-crRNA1 complexes that induced indel mutations are located within the yellow marked locus

\section{Abbreviations}

Cas: CRISPR-associated protein; CRISPR: Clustered regularly interspaced short palindromic repeats; crRNA: CRISPR RNA; MLO: Mildew resistance locus O; PEG: Polyethylene glycol; RNP: Ribonucleoprotein; sgRNA : Single guide RNA

\section{Acknowledgments}

We are grateful to Professor Byoung-Cheorl Kang of Seoul National University and the Vegetable Breeding Research Center for sharing two pepper cultivars, CM334 and Dempsey. We appreciate the technical contributions provided by Dr. Jiyeon Kweon, Ms. Ha Rim Shin, Professor Yongsub Kim, and Ms. Min Kyung Choi. We thank Ms. Hyunah Lee of the Central Laboratory for Kangwon National University for the help with the LSM880 microscope. We appreciate the imaging support by the Korea Basic Science Institute (KBSI) National Research Facilities \& Equipment Center (NFEC) grant funded by the Korea government (Ministry of Education) (2019R1A6C1010006)

\section{Authors' contributions}

HK devised and performed the project. JC and KHW performed and assisted with the experiments. HK, JC, and KHW analyzed the results. HK wrote the manuscript. All authors have approved the manuscript.

\section{Funding}

This work was supported by grants from the New Breeding Technologies Development Program [Project No. PJ01477602], Rural Development Administration (RDA), Republic of Korea, and the Basic Science Research Program of the National Research Foundation of Korea funded by the Ministry of Education, Science and Technology [Grant No. 2018R1A2B6006233] to HK, and by the National Research Foundation of Korea and Center for Women in Science, Engineering, and Technology [Grant No. WISET-2019-674ho] to JC. The funders had no role in the design of the study and collection, analysis, and interpretation of data and in writing the manuscript.

\section{Availability of data and materials}

The datasets used and/ or analyzed during the current study are available from the corresponding author upon reasonable request. The pepper cultivars should be requested from the Vegetable Breeding Research Center (VBRC).

Ethics approval and consent to participate Not applicable.

Consent for publication Not applicable.

Competing interests

All authors declare that they have no competing interests. 
Received: 19 May 2020 Accepted: 23 September 2020

Published online: 01 October 2020

\section{References}

1. Jinek M, Chylinski K, Fonfara I, Hauer M, Doudna JA, Charpentier E. A programmable dual-RNA-guided DNA endonuclease in adaptive bacterial immunity. Science. 2012;337:816-21.

2. Ran FA, Cong L, Yan WX, Scott DA, Gootenberg JS, Kriz AJ, et al. In vivo genome editing using Staphylococcus aureus Cas9. Nature. 2015;520:186-91.

3. Price AA, Sampson TR, Ratner HK, Grakoui A, Weiss DS. Cas9-mediated targeting of viral RNA in eukaryotic cells. Proc Natl Acad Sci U S A. 2015;112: 6164-9

4. Muller M, Lee CM, Gasiunas G, Davis TH, Cradick TJ, Siksnys V, et al. Streptococcus thermophilus CRISPR-Cas9 systems enable specific editing of the human genome. Mol Ther. 2016;24:636-44.

5. Kim E, Koo T, Park SW, Kim D, Kim K, Cho H-Y, et al. In vivo genome editing with a small Cas9 orthologue derived from Campylobacter jejuni. Nat Commun. 2017:8:14500.

6. Wang $H$, Yang $H$, Shivalila CS, Dawlaty MM, Cheng AW, Zhang F, et al. Onestep generation of mice carrying mutations in multiple genes by CRISPR/ Cas-mediated genome engineering. Cell. 2013;153:910-8.

7. Li D, Qiu Z, Shao Y, Chen Y, Guan Y, Liu M, et al. Heritable gene targeting in the mouse and rat using a CRISPR-Cas system. Nat Biotechnol. 2013;31:6813.

8. Shan Q, Wang Y, Li J, Zhang Y, Chen K, Liang Z, et al. Targeted genome modification of crop plants using a CRISPR-Cas system. Nat Biotechnol. 2013;31:686-8

9. Li J-F, Norville JE, Aach J, McCormack M, Zhang D, Bush J, et al. Multiplex and homologous recombination-mediated genome editing in Arabidopsis and Nicotiana benthamiana using guide RNA and Cas9. Nat Biotechnol. 2013;31:688-91.

10. Nekrasov V, Staskawicz B, Weigel D, Jones JDG, Kamoun S. Targeted mutagenesis in the model plant Nicotiana benthamiana using Cas9 RNAguided endonuclease. Nat Biotechnol. 2013;31:691-3.

11. Wang Y, Cheng X, Shan Q, Zhang Y, Liu J, Gao C, et al. Simultaneous editing of three homoeoalleles in hexaploid bread wheat confers heritable resistance to powdery mildew. Nat Biotechnol. 2014;32:947-51.

12. Cong L, Ran FA, Cox D, Lin S, Barretto R, Habib N, et al. Multiplex genome engineering using CRISPR/Cas systems. Science. 2013;339:819-23.

13. Mali P, Yang L, Esvelt KM, Aach J, Guell M, DiCarlo JE, et al. RNA-guided human genome engineering via Cas9. Science. 2013;339:823-6.

14. Cho SW, Kim S, Kim JM, Kim J-S. Targeted genome engineering in human cells with the Cas9 RNA-guided endonuclease. Nat Biotechnol. 2013;31:2302.

15. Zetsche B, Gootenberg JS, Abudayyeh OO, Slaymaker IM, Makarova KS, Essletzbichler P, et al. Cpf1 is a single RNA-guided endonuclease of a class 2 CRISPR-Cas system. Cell. 2015;163:759-71.

16. Schunder E, Rydzewski K, Grunow R, Heuner K. First indication for a functional CRISPR/Cas system in Francisella tularensis. Int J Med Microbiol. 2013:303:51-60.

17. Hur JK, Kim K, Been KW, Baek G, Ye S, Hur JW, et al. Targeted mutagenesis in mice by electroporation of Cpf1 ribonucleoproteins. Nat Biotechnol. 2016; 34:807-8.

18. Kim H, Kim S-T, Ryu J, Kang B-C, Kim J-S, Kim S-G. CRISPR/Cpf1-mediated DNA-free plant genome editing. Nat Commun. 2017;8:14406.

19. Xu R, Qin R, Li H, Li D, Li L, Wei P, et al. Generation of targeted mutant rice using a CRISPR-Cpf1 system. Plant Biotechnol J. 2017;15:713-7.

20. Kleinstiver BP, Pattanayak V, Prew MS, Tsai SQ, Nguyen NT, Zheng Z, et al. High-fidelity CRISPR-Cas9 nucleases with no detectable genome-wide offtarget effects. Nature. 2016;529:490-5.

21. Slaymaker IM, Gao L, Zetsche B, Scott DA, Yan WX, Zhang F. Rationally engineered Cas9 nucleases with improved specificity. Science. 2016;351:848.

22. Fu Y, Sander JD, Reyon D, Cascio VM, Joung JK. Improving CRISPR-Cas nuclease specificity using truncated guide RNAs. Nat Biotechnol. 2014;32: 279-84

23. Kim S, Koo T, Jee H-G, Cho H-Y, Lee G, Lim D-G, et al. CRISPR RNAs trigger innate immune responses in human cells. Genome Res. 2018;28:367-73.

24. Woo JW, Kim J, Kwon SI, Corvalan C, Cho SW, Kim H, et al. DNA-free genome editing in plants with preassembled CRISPR-Cas9 ribonucleoproteins. Nat Biotechnol. 2015;33:1162-4.
25. Miao J, Guo D, Zhang J, Huang Q, Qin G, Zhang X, et al. Targeted mutagenesis in rice using CRISPR-Cas system. Cell Res. 2013;23:1233-6.

26. Park SH, Morris JL, Park JE, Hirschi KD, Smith RH. Efficient and genotypeindependent Agrobacterium-mediated tomato transformation. J Plant Physiol. 2003;160:1253-7.

27. Cortina C, Culiáñez-Macià FA. Tomato transformation and transgenic plant production. Plant Cell Tissue Organ Cult. 2004;76:269-75.

28. Klein TM, Harper EC, Svab Z, Sanford JC, Fromm ME, Maliga P. Stable genetic transformation of intact Nicotiana cells by the particle bombardment process. Proc Natl Acad Sci U S A. 1988;85:8502-5.

29. Krügel T, Lim M, Gase K, Halitschke R, Baldwin IT. Agrobacterium-mediated transformation of Nicotiana attenuata, a model ecological expression system. Chemoecology. 2002;12:177-83.

30. Kim S, Park M, Yeom S-I, Kim Y-M, Lee JM, Lee H-A, et al. Genome sequence of the hot pepper provides insights into the evolution of pungency in Capsicum species. Nat Genet. 2014;46:270-8.

31. Qin C, Yu C, Shen Y, Fang X, Chen L, Min J, et al. Whole-genome sequencing of cultivated and wild peppers provides insights into Capsicum domestication and specialization. Proc Natl Acad Sci U S A. 2014;111:5135-40.

32. Kim H, Lim J. Leaf-induced callus formation in two cultivars: hot pepper "CM334" and bell pepper "Dempsey". Plant Signal Behav. 2019;14:1604016.

33. Cerkauskas RF, Buonassisi A. First report of powdery mildew of greenhouse pepper caused by Leveillula taurica in British Columbia, Canada. Plant Dis. 2003;87:1151.

34. Elad Y, Messika Y, Brand M, David DR, Sztejnberg A. Effect of microclimate on Leveillula taurica powdery mildew of sweet pepper. Phytopathology. 2007;97:813-24

35. Consonni C, Humphry ME, Hartmann HA, Livaja M, Durner J, Westphal L, et al. Conserved requirement for a plant host cell protein in powdery mildew pathogenesis. Nat Genet. 2006;38:716-20.

36. Acevedo-Garcia J, Gruner K, Reinstadler A, Kemen A, Kemen E, Cao L, et al. The powdery mildew-resistant Arabidopsis mlo2 mlo6 mlo12 triple mutant displays altered infection phenotypes with diverse types of phytopathogens. Sci Rep. 2017;7:9319.

37. Kim DS, Hwang BK. The pepper MLO gene, CaMLO2, is involved in the susceptibility cell-death response and bacterial and oomycete proliferation. Plant J. 2012;72:843-55.

38. Zheng Z, Nonomura T, Appiano M, Pavan S, Matsuda Y, Toyoda H, et al. Loss of function in Mlo orthologs reduces susceptibility of pepper and tomato to powdery mildew disease caused by Leveillula taurica. PLoS One. 2013;8:e70723.

39. Park J, Bae S, Kim J-S. Cas-designer: a web-based tool for choice of CRISPRCas9 target sites. Bioinformatics. 2015;31:4014-6. https://doi.org/10.1093/ bioinformatics/btv537.

40. Jeong SY, Ahn H, Ryu J, Oh Y, Sivanandhan G, Won K-H, et al. Generation of early-flowering Chinese cabbage (Brassica rapa spp. pekinensis) through CRISPR/Cas9-mediated genome editing. Plant Biotechnol Rep. 2019;13:4919. https://doi.org/10.1007/s11816-019-00566-9.

41. Xu J, Kang B-C, Naing AH, Bae S-J, Kim J-S, Kim H, et al. CRISPR/Cas9mediated editing of 1-aminocyclopropane-1-carboxylate oxidase1 enhances Petunia flower longevity. Plant Biotechnol J. 2020;18:287-97.

42. Cai Y, Chen L, Liu X, Guo C, Sun S, Wu C, et al. CRISPR/Cas9-mediated targeted mutagenesis of $\mathrm{GmFT} 2 \mathrm{a}$ delays flowering time in soya bean. Plant Biotechnol J. 2018;16:176-85.

43. Liang Z, Zhang K, Chen K, Gao C. Targeted mutagenesis in Zea mays using TALENs and the CRISPR/Cas system. J Genet Genomics. 2014;41:63-8.

44. Brooks C, Nekrasov V, Lippman ZB, Van Eck J. Efficient gene editing in tomato in the first generation using the clustered regularly interspaced short palindromic repeats/ CRISPR-associated 9 system. Plant Physiol. 2014;166:1292-7.

45. Wang S, Zhang S, Wang W, Xiong X, Meng F, Cui X. Efficient targeted mutagenesis in potato by the CRISPR/Cas9 system. Plant Cell Rep. 2015;34:1473-6.

46. Tian S, Jiang L, Gao Q, Zhang J, Zong M, Zhang H, et al. Efficient CRISPR/ Cas9-based gene knockout in watermelon. Plant Cell Rep. 2017;36:399-406.

47. Jie E-Y, Kim S-W, Jang H-R, In D-S, Liu J-R. Myo-inositol increases the plating efficiency of protoplast derived from cotyledon of cabbage (Brassica oleracea var. capitata). J Plant Biotechnol. 2011;38:69-76.

48. Park J, Lim K, Kim J-S, Bae S. Cas-analyzer: an online tool for assessing genome editing results using NGS data. Bioinformatics. 2017;33:286-8.

\section{Publisher's Note}

Springer Nature remains neutral with regard to jurisdictional claims in published maps and institutional affiliations. 